\title{
ASPECTOS DA REINSERÇÃO SOCIAL DO OSTOMIZADO*
}

\author{
Luciana Maria Boccardo** \\ Sueli Aparecida Nogueira ${ }^{\star \star}$ \\ Ednilza Ribeiro dos Santos**

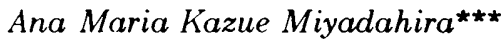 \\ Vera Lucia Conceição de Gouveia Santos ${ }^{\star \star \star \star}$
}

BOCCARDO, L.M. et al Aspectos da reinserção social do ostomizado. Rev.Esc.Enf.USP, v.29, n.1, p.59-71, abr. 1995 .

A reinserção social é um desafio para o ostomizado e uma preocupação para a equipe interdisciplinar. Sendo assim, esse estudo visa verificar as dificuldades apre. sentadas pelos ostomizados atendidos em dois serviços ambulatoriais da cidade de São Paulo, quanto ao retorno às atividades cotidianas da fase pré-doença (domésticas, de lazer, trabalho e sexuais). Através da análise dos resultados obtidos nas entrevistas com 15 ostomizados, subdivididos em 3 grupos conforme o tempo de pósoperatório, pode-se verificar que a maior parte da clientela não retornou totalmente ou retornou apenas parcialmente às atividades de vida diária, principalmente as sexuais. Dentre as justificativas referidas pelos 3 grupos, destacam-se os problemas físicos, a insegurança e a inadequação do dispositivo utilizado.

UNITERMOS: Reabilitação. Assistência ao ostomizado. Autocuidado. Estomaterapia.

\section{INTRODUÇÃO}

O processo de reinserção social de pessoas portadoras de ostomias constitui o foco central do cuidado prestado pela equipe multidisciplinar inserido no contexto de reabilitação.

A reinserção social é um desafio para o ostomizado na medida que a confecção de um estoma representa e supõe uma agressão à integridade físi-

\footnotetext{
* Trabalho apresentado no 42 Congresso Brasileiro de Colo-proctologia, no 45o Congresso Brasileiro de Enfermagem e premiado durante o I Simpósio Internacional de Estomaterapia, II Fncontro Nacional de Estomaterapia e V Curso de Estomaterapia.

** Nunos do Curso de (iraduação em Enfermagem da LISP.

*** Enfermeira. Professora Doutora do Departamento de Enfermagem Médico-Cirúrgica da Escola de Enfermagem da USP.

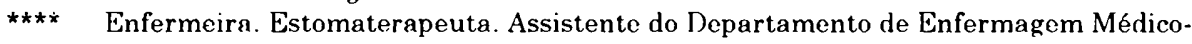
Cirúrgica da Escola de Enfermagem da USP.
} 
ca e psíquica com severas repercussões nos planos da imagem corporal e autoconceito ${ }^{5}$. Shilder citado por TOMASELLI; MORIN ${ }^{14}$, define imagem corporal como a percepção que o indivíduo forma do seu próprio corpo. Assim, o significado do estoma para o indivíduo, passa por diversas fases até a reconstrução de uma nova identidade, com a incorporação dessa nova situação. COHEN $^{4}$, descreve como primeiro estágio (fase pós operatória mais precoce) aquele em que o estoma é percebido pelo paciente como uma doença, algo estranho e sem controle. No segundo estágio, o estoma constitui um "it", isto é, tanto a identidade do indivíduo como a do estoma não são claras para o paciente. Já no terceiro estágio, o estoma passa a ter uma identidade, mesmo que de doença. Ele é assim, uma parte legítima do ostomizado.

O estoma é, portanto, muitas vezes o custo da saúde para o indivíduo, exigindo que ele desenvolva tal processo de reconstrução de identidade pessoal e reformulação de sua imagem corporal. Somente desta forma conseguirá atingir um nível adequado de reabilitação, voltando a assumir suas atividades pré-operatórias e pré-doença, mesmo com dificuldades, o mais próximo do normal possível.

Para tanto, o papel do enfermeiro é fundamental como elemento de uma equipe, na medida que o auxilia a tornar-se independente através da reaquisição da sua autonomia para manter sua dignidade e voltar a desenvolver a sua auto-estima.

Diversos estudos têm sido realizados na busca da melhor definição de qualidade de vida e reabilitação do ostomizado, incluindo como fator essencial a reinserção social. No Brasil, entretanto, a estomaterapia constitui uma especialidade ainda recente e a carência de estudos nessa área associada a inserção desse tema nas escolas de graduação, principalmente em São Paulo, motivou a realização desse trabalho, em continuidade a estudo ànterior, (NO. GUEIRA et al ${ }^{9}$ ) buscando nesse caso aprofundar a identificação das dificuldades relacionadas ao autocuidado na esfera social.

Constituíram, portanto, seus objetivos: verificar as dificuldades apresentadas pelos ostomizados quanto ao retorno às atividades domésticas, de lazer, de trabalho e sexuais e comparar as respostas apresentadas pelos ostomizados quanto ao processo reabilitatório, em diferentes períodos da fase pós-operatória tardia.

\section{METODOLOGIA}

A população foi constituída de 45 pacientes portadores de estomas urinários e intestinais em seguimento ambulatorial, atendidos em dois serviços governamentais da cidade de São Paulo. Apesar da população ser heterogênea, esses serviços têm funções similares: prestar assistência 
multidisciplinar a ostomizados incluindo a seleção e distribuição de dispositivos. Fazem parte desta equipe, em ambos os serviços, enfermeiras estomaterapeutas.

Para serem incluidos no estudo, os pacientes deveriam aceitar participação no estudo; ter idade superior a 18 anos e estar em condições de oferecer informações através da entrevista.

Os pacientes foram distribuídos em três grupos, conforme a fase pósoperatória tardia, quais sejam:

- Grupo A: 15 pacientes que tiveram alta hospitalar em até três meses, de operações que resultaram na confecção de estoma;

- Grupo B: 15 pacientes que tiveram alta hospitalar no período compreendido entre três a doze meses;

- Grupo C: 15 pacientes que tiveram alta hospitalar acima de doze meses.

Os dados foram coletados em outubro e novembro de 1992, através de entrevista, utilizando um formulário claborado especialmente para este estudo, composto de questões abertas e fechadas, distribuídas em 4 ítens básicos:

- dados de identificação;

- dados acerca da hospitalização;

- dados sobre o retorno às atividades de vida diária e

- dificuldades referidas após a alta hospitalar.

As entrevistas foram individuais e realizadas por 12 alunos que, no período citado cursava a disciplina Enfermagem Médico-Cirúgica da Escola de Enfermagem da Universidade de São Paulo.

\section{RESULTADOS E DISCUSSÃO}

Os resultados são distribuídos em três subitens: caracterização da população, dados acerca da hospitalização (os quais já se encontram descritos em NOGUEIRA et al $^{9}$, estabelecendo-se aqui apenas uma revisão) e dados sobre atividades após a alta hospitalar.

\section{Caracterização da população}

A população em estudo foi caracterizada quanto a idade, sexo, estado civil e número de dependentes, o que pode ser observado na Tabela 1. 
Tabela 1 - Número de ostomizados nos 3 grupos, segundo sexo, idade, estado civil e número de dependentes. São Paulo, 1992.

\begin{tabular}{|c|rr|rrr|rrr|rrr|}
\hline & \multicolumn{2}{|c|}{ Sexo } & \multicolumn{3}{c|}{ Idade } & \multicolumn{2}{|c|}{ Estado civil } & \multicolumn{2}{|c|}{$\mathbf{N}^{\mathbf{2}}$ de dependentes } \\
\hline GRUPOS & M & F & $\mathbf{1 8 - 3 8}$ & $\mathbf{3 8 - 5 8}$ & $\mathbf{5 8 - 7 8}$ & sol. & cas. & viu. & $\mathbf{0}$ & até 2 & $\mathbf{3}$ e + \\
\hline A & 8 & 7 & 7 & 4 & 4 & 10 & 4 & 1 & 9 & 9 & 1 \\
B & 9 & 6 & 3 & 7 & 7 & 9 & 3 & 3 & 6 & 6 & 3 \\
C & 6 & 9 & 2 & 4 & 9 & 8 & 3 & 4 & 8 & 5 & 2 \\
& & & & & & & & & & & \\
\hline TOTAL & $\mathbf{2 3}$ & $\mathbf{2 2}$ & $\mathbf{1 2}$ & $\mathbf{1 5}$ & $\mathbf{1 8}$ & $\mathbf{2 7}$ & $\mathbf{1 0}$ & $\mathbf{8}$ & $\mathbf{2 3}$ & $\mathbf{1 6}$ & $\mathbf{6}$ \\
\hline
\end{tabular}

Os dados da Tabela 1 mostram uma distribuição eqüitativa quanto ao sexo, no total e internamente nos 3 grupos. Houve predomínio global da faixa etária de 58 a 78 anos, sendo que a menor incidência esteve presente para os pacientes em faixa etária menor (18 a 38 anos) que, ao ser somada com aquela entre 38 e 58, resulta num total de 21 (mais da metade) na faixa até 58 anos, ainda produtiva em nosso país. Segundo a idade, a distribuição nos grupos foi diferenciada para o grupo C onde era esperada idade média maior em função do tempo em que estão ostomizados, obtendo-se a maioria operada há mais de 10 anos.

Quanto ao estado civil, observa-se que a maioria dos pacientes é casada (27). Isso tem importância no ponto de vista psicossocial e mesmo econômico, já que esta situação pode estar relacionada aos problemas resultantes da ostomia, nas esferas conjugal, sexual e de trabalho $0^{6,10,15}$.

$\Lambda$ Tabela 1 mostra ainda que a maioria dos pacientes não tem dependentes (23), o que levaria a pensar numa diminuição das preocupações dessa clientela com a questão trabalho. Entretanto, ao somar o número daqueles que têm um e mais dependentes, nota-se que totalizam 22 pacientes (quase a metade), sendo 6 deles com 3 e mais dependentes. Relacionando esses dados com o total de pacientes na faixa etária de 18 a 58 anos, numa somatória de 27 , e que estariam em idade produtiva, a questão financeira pode ser um fator determinante no processo de reinserção social.

\section{Dados acerca da hospitalização}

A população estudada foi também caracterizada quanto a:

- causas das operações, onde 35 apresentaram as neoplasias dos vários seguimentos intestinais e urinários; 
- tipos das operações, sobressaindo-se as amputações de reto (22) e colectomias parciais e totais (16) e

- tipo de orientação recebida durante a hospitalização, obtendo-se, como maior número de respostas a higiene e troca da bolsa(27).

Ao considerar-se a faixa etária predominante neste estudo (Tabela 1), verifica-se a compatibilidade entre a maior incidência das neoplasias e a maior parte dos ostomizados apresentarem idade superior a 40 anos, característica esta tida não só como fator de risco para o aparecimento dos cânceres como sua própria incidência aumentada a partir desta idade ${ }^{6.7 .10,12.15}$

$O$ diagnóstico de câncer se apresenta como um agravante a mais no processo de reabilitação, e portanto, podendo dificultar a reinserção social. Além de todas as dificuldades inerentes à adaptação à nova condição de vida do ostomizado, esse diagnóstico é visto erroneamente como sinônimo de mor$\mathrm{te}^{15}$.

O predomínio das operações de amputação de reto e colectomias (38), está diretamente relacionado às causas mais freqüentes para a confecção de estomas, que são as neoplasias, principalmente in testinais.

No tocante ao tipo de orientação oferecida durante a hospitalização, diversos autores apontam que e fundamental para o êxito do processo de reabilitação, a implementação de uma assistência sistematizada precoce, desde a fase pré-operatória e que incluirá orientações capazes de proporcionar ao ostomizado segurança no momento da alta hospitalar $2,8,12,15,16,17$, certamente muito mais abrangente e profunda do que aquelas citadas pela maioria dos pacientes, relacionadas basicamente a limitados aspectos de cuidado local.

\section{Dados sobre atividades desenvolvidas após a alta hospitalar.}

Os ostomizados foram interrogados sobre o retorno às atividades domésticas, de lazer, sexuais e de trabalho, bem como suas dificuldades e justificativas.

Dos 45 entrevistados, 21 não retornaram às suas atividades domésticas, sendo que, destes, o maior número encontra-se no grupo A (10). Pode-se propor como explicação, o pouco tempo transcorrido desde a operação; enquanto para os grupos $\mathrm{B}$ e $\mathrm{C}$, a distribuição foi de 8 e 3 , respectivamente. Dentre os 24 que afirmaram ter retornado, 12 relataram ser parcialmente. Destes, 7 'estão no grupo C.

As justificativas das dificuldades para o não retorno e retorno parcial estão apresentadas na Tabela 2. 
Tabela 2 - Número de respostas dos 33 pacientes nos 3 grupos, segundo justificativas para o não retorno e retorno parcial às atividades do mésticas. São Paulo, 1992.

\begin{tabular}{|c|ccc|ccc|c|}
\hline GRUPOS & \multicolumn{3}{|c|}{$\begin{array}{c}\text { RETORNO } \\
\text { PARCIAL }\end{array}$} & \multicolumn{3}{|c|}{ NÃO RETORNO } & \\
\hline JUSTIFICATIVA & A & B & C & A & B & C & TOTAL \\
\hline Problemas físicos $^{\star}$ & 3 & 1 & 5 & 7 & 6 & 1 & 23 \\
\hline $\begin{array}{c}\text { Inadequação da } \\
\text { Bolsa }\end{array}$ & 1 & - & 3 & 2 & - & - & 6 \\
\hline Tem quem o auxilie & - & - & - & 1 & 2 & 2 & 5 \\
\hline TOTAL & 4 & 1 & 8 & 10 & 8 & 3 & 34 \\
\hline
\end{tabular}

* dor, deiscência

Das 34 respostas dos pacientes que não retornaram e dos que retornaram parcialmente às atividades domésticas, 23 citações relacionamse aos problemas físicos.

Quanto ao retorno parcial, verifica-se que a maioria das respostas foi mencionada por pacientes do grupo $C$ (8) que, conforme as justificativas, queixam-se de problemas físicos (5) e inadequação da bolsa (3). Esses dados chamam a atenção, uma vez que o tempo mínimo de confeç̧ão do estoma desse grupo é de um ano. Porém, é sabido que esse grupo é constituído por uma maioria (9) na faixa etária entre 58 a 78 anos, o que sugere a maior probabilidade de problemas físicos que podem ou não estar associados à operação e doença de base. Segundo RUBIN; DEVLIN" ${ }^{\prime \prime}$ cerca de $80 \%$ dos colostomizados (que constituem a maioria dos pacientes deste estudo), possuem problemas de saúde não associados à doença de base como artrites e reumatismos ocasionando capacidades funcionais prejudicadas. Para os ileostomizados, no entanto, existem alterações conseqüentes não só às doenças inflamatórias mas também ao próprio estoma, como as colelitíases e urolitíases $^{11,13}$.

Quanto às 3 respostas para inadequação da bolsa podem estar associadas à qualidade do equipamento utilizado por estes pacientes seja por falta de orientação ou pela própria inexistência ou insuficiência do dispositivo de 
melhor qualidade. Neste sentido, cabe esclarecer que na época em que os dados foram coletados, faltavam dispositivos nos referidos serviços públiços de assistência.

No que tange ao não retorno, os problemas físicos foram igualmente os mais referidos (14) e, destes, 13 pertencem aos grupos A e B. Isso pode estar ligado à fase de convalescença pós-cirúrgica e à submissão a tratamentos adjuvantes como a quimioterapia e radioterapia, o que condiz com as causas mais freqüentes para as ostomias, ou seja, as neoplasias ${ }^{7,8,15,16}$.

$\lceil$ Ao observar esses resultados constata-se que, num período inicial, os problemas físicos superam os de ordem psicologica ou social, retardando, ássim, o retorno às atividades domésticas, sobretudo, se o trabalho demandar esforço físico demasiado, o que poderia resultar em complicações como hérni. as paracolostômicas e prolapso ${ }^{12,5,7}$.

Os dados levantados também se relacionam às atividades de lazer, tendo se verificado que 17 pacientes não retornaram a essas atividades. A distribuição desses pacientes foi eqüitativa entre os grupos $\mathrm{A}, \mathrm{B}$ e $\mathrm{C}$, sendo 6,6 e 5 , respectivamente.

Dentre os 28 que retornaram a essas atividades, 15 referem ter sido parcialmente. Destes, mais da metade pertencem ao grupo C. Assim, somando-se os números de quem não retornou aqueles que retornaram parcialmente têm-se que apenas 2 pacientes do grupo C retornaram totalmente as atividades de lazer. As justificativas dos pacientes para o não retorno e retorno parcial às atividades de lazer encontram-se na Tabela 3

Tabela 3 - Número de respostas dos 32 pacientes dos 3 grupos, segundo justificativas para o não retorno e retorno parcial às atividades de lazer São Paulo, 1992.

\begin{tabular}{|c|ccc|ccc|c|}
\hline GRUPOS & \multicolumn{3}{|c|}{$\begin{array}{c}\text { RETORNO } \\
\text { PARCIAL }\end{array}$} & \multicolumn{2}{|c|}{ NÃO RETORNO } & \\
\hline JUSTIFICATIVA & A & B & C & A & B & C & TOTAL \\
\hline Insegurança & 3 & 4 & 5 & 2 & 3 & 3 & 20 \\
\hline Problemas físicos* $^{*}$ & 3 & - & - & 5 & 3 & 1 & 12 \\
\hline $\begin{array}{c}\text { Dificuldades para } \\
\text { higienização da } \\
\text { Bolsa }\end{array}$ & - & - & - & 1 & 2 & 1 & 4 \\
\hline Vergonha & - & 1 & - & - & 1 & 1 & 3 \\
\hline TOTAL & 6 & 5 & 5 & 8 & 9 & 6 & 39 \\
\hline
\end{tabular}

*dificuldades para sentar. fraqueza, operaçăo recente. 
Observa-se nessa Tabela que o número de respostas relacionadas à insegurança predomina sobre as demais justificativas. Isso pode estar ligado à qualidade do dispositivo que para os ostomizados determina, em grande parte, o seu bem-estar social. Referente à distribuição das respostas sobre insegurança, nota-se que novamente houve um número significativo de citações por parte de pacientes do grupo C (5 para o retorno parcial e 3 para o não retorno). Esta constatação leva a inferência de que a insegurança independe do tempo transcorrido após a operação. A ostomia é por si mesma um agente agressivo que opera através da sensação de perda de segurança e integrida$\mathrm{de}^{2,5,8}$

Segundo BURCKHARDT et $\mathrm{al}^{3}$, um estudo feito com pacientes portadores de doenças crônicas, entre eles ostomizados - considerados saudáveis com seqüela crônica - dentre as áreas apontadas como indicadores para a qualidade de vida estão a independência, habilidade em se cuidar e senso de segurança. Já para RUBIN; DEVLIN" ${ }^{11} 20 \%$ de ostomizados (entre colostomizados e ileostomizados) restringem o retorno às atividades sociais por medo principalmente de funcionamento do estoma e ausência de banheiros adequados, o que se pode traduzir como insegurança.

Além desses fatores apontados, a insegurança pode estar também relacionada a dificuldade de higienizar a bolsa, bem como ao uso de dispositivos inadequados, conforme já citados e discutidos anteriormente. Essas constatações justificam a necessidade de uma assistência sistematizada, interdisciplinar, precoce e institucionalizada.

Em relação ao retorno ao trabalho, obteve-se que 27 dos 45 entrevistados, não retornaram a essas atividades. Dentre os 27 , a maioria absoluta (24) pertence aos grupos A e B (14 e 10, respectivamente). Dos 18 pacientes que retornaram, 13 referem ter sido com dificuldades.

Diante disso, parece ter havido uma distribuição coerente dos pacientes entre os grupos, ou seja, esperava-se realmente que um maior número de ostomizados pertencentes aos grupos $\mathrm{B}$, sobretudo o grupo $\mathrm{A}$, não tivessem retornado às atividades de trabalho, o que se explica pelo tempo máximo e mínimo de pos-operatório para esses grupos.

Quanto aos pacientes que retornaram com dificuldades, a maioria pertence ao grupo $\mathrm{C}$, o que pode estar diretamente relacionado com o fator idade, entre 58 e 78 anos (Tabela 1 ).

As justificativas para o não retorno às atividades de trabalho encontram-se na Tabela 4 
Tabela 4 - Número de respostas dos 27 pacientes dos 3 grupos, segundo justificativas para o não retorno e retorno parcial às atividades de trabalho. São Paulo, 1992.

\begin{tabular}{|c|c|c|c|c|}
\hline JUSTIFICATIVAS & A & & C & TOTAL \\
\hline Problemas físicos* & 8 & 4 & - & 12 \\
\hline Aposentadoria & 2 & 2 & 2 & 6 \\
\hline Licença Médica & 2 & 2 & 1 & 5 \\
\hline $\begin{array}{l}\text { Problemas com a } \\
\text { Bolsa }\end{array}$ & 1 & 1 & 2 & 4 \\
\hline TOTAL & 13 & 9 & 5 & 27 \\
\hline
\end{tabular}

* dificuldades para sentar, fraqueza, operação recente.

Conforme a Tabela 4, as justificativas mais citadas (12) como causas do não retorno às atividades de trabalho foram os problemas físicos que, como já visto neste estudo, têm sido um fator determinante para a volta às atividades desenvolvidas na fase pré-doença e pré-cirúrgica.

Quanto aos grupos pode-se dizer que houve uma distribuição sugestivamente coerente, pois das 12 respostas referentes aos problemas físicos, mais da metade (8) estão no grupo A. De fato os pacientes desse grupo, nesse período, podem ainda não ter superado a problemática de ordem física, como afirmam vários autores ${ }^{3,5,8,12}$. Além disso, para os pacientes dos grupos A e $B$, os controles ambulatoriais, a que são submetidos nessa fase, são freqüentes e a alta incidência de neoplasias gera, muitas vezes, a necessidade dos tratamentos adjuvantes já mencionados (radio e quimioterapia), que implicam em visitas constantes, até diárias, aos Hospitais. Esta sugestão já foi considerada quanto ao não retorno às atividades domésticas (Tabela 2).

É necessário também considerar as características da clientela atendida nos serviços utilizados como campo para a coleta de dados, provenientes de diversos locais, com trabalhos de diferentes naturezas, entre os quais, lavoura, construção civil, estiva e outros que demandam elevados graus de esforços físicos. 
Quanto à justificativa de ser aposentado (6 respostas), ressalta-se que a clientela desse estudo é de baixa renda e, portanto, o fato de ser aposentada não explicaria o não retorno ao trabalho, a não ser que associada ao fator idade. RUBIN; DEVLIN ${ }^{11}$, mencionam que 20 a $90 \%$ dos colostomizados não retornam ao trabalho por terem suas capacidades afetadas devido à idade $\mathrm{e}$ doenças crônicas associadas.

Embora em pequeno número (4), verifica-se que os problemas com a bolsa ainda são citados e que poderiam ter sido resolvidos com o seguimento do paciente através de um Programa Assistencial, em nível público.

Diante das justificativas apresentadas para o não retorno às atividades de trabalho, corrobora-se mais uma vez a importância da equipe interdisciplinar no processo de reabilitação do ostomizado, pois a deficiência na "recolocação" no trabalho, quando necessária, pode indicar as limitações da atuação do Serviço Social.

A grande maioria dos ostomizados entrevistados não retornou às atividades sexuais (31), com uma distribuição decrescente em relação ao tempo de pós-operatório. Assim, têm-se 13 no grupo A, 10 em B e 8 em C. Dos 14 que afirmaram ter voltado a essa atividade, 9 referiram ter sido com dificuldades.

Conforme os dados anteriores, há um número significativo de pacientes (27) em fase sexualmente ativa, isto é, entre 18 e 58 anos (Tabela 1). Esse dado quando relacionado ao número daqueles que não retornou às atividades sexuais, mostra o quanto a confecção de um estoma interfere nesse tipo de atividade.

$\Lambda$ atividade sexual ć um fator preocupante na vida do ostomizado. Muitos deles não praticam sexo por sentirem medo, vergonha, tristeza. Esses pacientes temem a reação do (a) parceiro (a), pois estes podem sentir-se inibidos ao verem o dispositivo coletor, terem nojo e rejeitarem a prática sexual $^{15}$.

As justificativas para o não retorno às atividades sexuais e para as dificuldades estão apresentadas na Tabela 5

Através dos dados da Tabela 5, observa-se que o maior número de respostas dos pacientes que retornaram às atividades sexuais com dificuldades, pertencem ao grupo C (5). Quanto às respostas relacionadas ao não retorno, verifica-se uma maioria (13) mencionada por pacientes do grupo A, o que parece compatível com o período de convalescença que esses pacientes atravessam. Nota-se ainda, que os números referentes ao grupos $\mathrm{B}$ e $\mathrm{C}$, quanto ao não retorno, são significativos. Isso mostra o quanto a atividade sexual é prejudicada pela confecção de um estoma, seja por problemas físicos, psíquicos como aqueles relacionados à auto-imagem (vergonha), a qualidade do dispositivo ou ainda pela rejeição do (a) parceiro (a). 
Tabela 5 - Número de respostas dos 40 pacientes nos 3 grupos, segundo as justificativas para o não retorno e retorno com dificuldades às ati vidades sexuais. São Paulo, 1992.

\begin{tabular}{|c|ccc|ccc|c|}
\hline GRUPOS & \multicolumn{3}{|c|}{$\begin{array}{c}\text { RETORNO } \\
\text { PARCIAL }\end{array}$} & \multicolumn{2}{c|}{$\begin{array}{c}\text { RETORNO COM } \\
\text { DIFICULDADE }\end{array}$} & \\
\hline JUSTIFICATIVA & A & B & C & A & B & C & TOTAL \\
\hline Problemas físicos * $^{*} 6$ & 5 & 2 & - & - & 3 & 16 \\
\hline Vergonha & 4 & - & 3 & - & - & - & 7 \\
\hline $\begin{array}{c}\text { Problemas com } \\
\text { dispositivo** }\end{array}$ & 1 & 2 & - & - & 2 & 1 & 6 \\
\hline $\begin{array}{c}\text { Não aceitação pelo } \\
\text { parceiro** }\end{array}$ & - & 1 & 1 & 2 & - & 1 & 5 \\
\hline Não tem parceiro & 2 & 2 & 2 & - & - & - & 6 \\
\hline TOTAL & 13 & 10 & 8 & 2 & 2 & 5 & 40 \\
\hline
\end{tabular}

*dor, impotência

** receio de vazamento

$\star \star \star$ preconceito do(a) parceiro(a)

Segundo RUBIN; DEVIIN", os problemas sexuais aparecem em mais de $67 \%$ dos colostomizados, homens e mulheres, com causas psicológicas e físicas. Já para os ileostomizados, embora em índices menores, ainda surgem em 0 a $30 \%$ dos casos. Em estudo feito por THOMPSON ${ }^{14}$, para $19 \%$ dos ileostomizados a função sexual parou após a cirurgia. Muitos indicaram como aspectos negativos nas relações com a família, nojo e conseqüente isolamento até a diminuição da espontaneidade e redução da nudez.

A realidade apontada tanto neste trabalho como na revisão de literatura, reflete a importância de uma assistência sistematizada e precoce, no intuito de reduzir ao máximo as dificuldades inerentes à condição de ostomizado, facilitando, portanto, um retorno mais satisfatório às atividades desenvolvidas nas fases pré-doença e pré-cirúrgica, ou seja, a reinserção social do portador de ostomia.

\section{CONCLUSÕES}

Os dados obtidos neste estudo permitiram as seguintes conclusões: 
- não houve um retorno satisfatório, segundo cada grupo às atividades diári as. Os resultados mostraram ainda que, mesmo com o passar do tempo, as dificuldades não são resolvidas. As justificativas estão relacionadas, principalmente, aos problemas físicos e ao dispositivo utilizado.

- ao comparar os três grupos, nota-se que a maioria absoluta dos ostomizados não retornou às atividades sexuais, havendo equivalência entre as respostas, nas diferentes fases do pós-operatório.

BOCCARDO, L.M. et al. Aspects of ostomized's social rehabilitation. Rev.Esc.Enf.USP, v.29, n.1. p.59-71, Apr. 1995.

The social rehabilitation represents a challenge to ostomy patient and a concern for health professional team. This study intends to verify the difficulties related to returning to the daily domestic, leisure, job and sexual activities showed by 45 ostomizeds after hospital discharge in different periods of late post operative. The data was obtained from patients assisted by two Outpatient Services of São Paulo city, through the interviews. The patients were selected by previous established criteria agreement and then subdivided in three groups as the post operative period. It's possible to verify that the majority of the clients didn't returned totally or returned only partially to the daily activities like before the disease or surgery, specially in the sexual area. They justified these difficulties by physical problems, insecurity and the use of inadequate appliances.

UNITERMS: Rehabilitation. Ostomy care. Self carc. Enterostomal therapy.

\section{REFERÊNCIAS BIBLIOGRÁFICAS}

J-AGUILLAR, I.F.G et al. Estomas digestivos. Rev. Enf. Cient., n. 110, p. 4-12, 1991.

2. BRECKMAN, B. Enfermeria del estoma. Madrid, Interamericana/McGraw-Hill, 1987.

'- BURCKHARDT, C.S. et al. Quality of life of adults with chronic illness: a psychometric study. Res. Nurs. Health, v. 12, p. 347-54, 1989.

4. COHEN, $A$. The stoma as a dominant symbol in the transition from sickness to health. WCET.e) v. 13, n. 3, p. 23-6, 1993

J GARCIA, A.M.M. et al. Atencion integral al paciente osto-mizado. Madrid, coloplast, 1992

)' GIMENO, A.M.M.; GAR(YIA, A.M.M. Propuesta para una valida reinsercion social de los enfermos colostomizados. s. ed., Valéncia, 1988. 
$\lambda$. HABR-GAMA, A. et al. Câncer do estômago e do intestino grosso. São Paulo. EDU/EDUSP, 1978.

8 - MAHONEY, Y.M. Guide to stomy nursing care. Boston, Little Brown, 1976.

9- NOGUEIRA, S.A.; SANTOS, E.R. dos; BO(CCARDO. L.M.; SANTOS, V.L.C. de G.;

MIYAD $\Lambda$ HIRA, A.M.K. Autocuidado do ostomizado: dificuldades percebidas após a alta hospitalar. Rev. Esc. Enf. USP, v.28, n. 3, p. , 1994.

10-RODRIGUES, J. et al. Problemática psicosocial del paciente ostomizado. Rev. Rol. Enf., v.11, n. 114, p. 233-33, 1988. I1. RUBIN, G. P.; DEVI.IN, H.B. The quality of life with a stoma. Br. J. Hosp. Med., v. 38, n.4, p.
$300-3,1987$.

2. SANTOS, V.L.C. de G. Reabilitação do ostomizado: em busca do ser saudável. Rev. Texto \& Contexto, v. 1, n. 2, p. 180-90, 1992. $\zeta_{\text {-THOMPSON, J.M. Quality of life after convencional ileostomy in NSW. WCET J., v. 10, n. 3, p. }}^{\text {8-14.1990. }}$

4 TOMASELLI, N.; MORIN, K.H. body image in patients with stomas: a critical review of the literature. J. ET. Nurs.. v. 18, n. 3, p. 95-9, 1991.

$\oint$ TRENTINI, M. et al. Vivendo com uma ostomia: um estudo preliminar. Rev. Gaucha Enf., v. 13, n. 2, p. 22-8, 1992 .

16. VUCKOVICH, V.C.; GRUBB, R.D. Care of the ostomy patient. Saint Louis, Mosby, 1977.

17 ZERBETTO, E.M. Roteiro para avaliação ao nivel de problemas do paciente colostomizado. São Paulo, 1981. Dissertação (mestrado) Escola de Enfermagem. Universidade de São Paulo. 